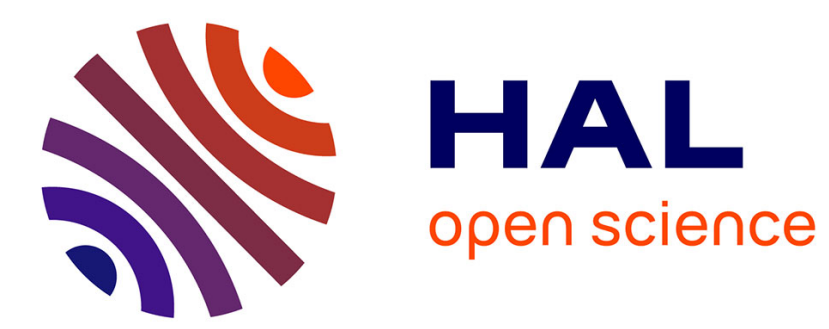

\title{
Broadcast Speedup in Vehicular Networks via Information Teleportation
}

Philippe Jacquet, Dalia Popescu, Bernard Mans

\section{To cite this version:}

Philippe Jacquet, Dalia Popescu, Bernard Mans. Broadcast Speedup in Vehicular Networks via Information Teleportation. LCN 2018 - 43rd Annual IEEE Conference on Local Computer Networks, Oct 2018, Chicago, United States. hal-01963430

\section{HAL Id: hal-01963430 \\ https://hal.inria.fr/hal-01963430}

Submitted on 21 Dec 2018

HAL is a multi-disciplinary open access archive for the deposit and dissemination of scientific research documents, whether they are published or not. The documents may come from teaching and research institutions in France or abroad, or from public or private research centers.
L'archive ouverte pluridisciplinaire HAL, est destinée au dépôt et à la diffusion de documents scientifiques de niveau recherche, publiés ou non, émanant des établissements d'enseignement et de recherche français ou étrangers, des laboratoires publics ou privés. 


\section{Broadcast Speedup in Vehicular Networks via Information Teleportation}

\author{
Philippe Jacquet \\ Nokia Bell Labs \\ France \\ philippe.jacquet@nokia-bell-labs.com
}

\author{
Dalia Popescu \\ Nokia Bell Labs \\ France \\ dalia-georgiana.popescu@nokia-bell-labs.com
}

\author{
Bernard Mans \\ Macquarie University \\ Sydney, Australia \\ bernard.mans@mq.edu.au
}

\begin{abstract}
The goal of this paper is to increase our understanding of the fundamental communication properties in urban vehicle-to-vehicle mobile networks by exploiting the selfsimilarity and hierarchical organization of modern cities. We use an innovative model called "hyperfractal" that captures the self-similarities of both the traffic and vehicle locations, and yet avoids the extremes of regularity and randomness.

We use analytical tools to derive matching theoretical upper and lower bounds for the information propagation speed in an urban delay tolerant network (i.e., a network that is disconnected at all time, and thus uses a store-carry-and-forward routing model). We prove that the average broadcast time behaves as $n^{1-\delta}$ (times a slowly varying function), where $\delta$ depends on the precise fractal dimension.

Furthermore, we show that the broadcast speedup is due in part to an interesting self-similar phenomenon, that we denote as information teleportation. This phenomenon arises as a consequence of the topology of the vehicle traffic, and triggers an acceleration of the broadcast time. We show that our model fits real cities where open traffic data sets are available. The study presents simulations that confirm the validity of the bounds in multiple realistic settings, including scenarios with variable speed.
\end{abstract}

\section{INTRODUCTION}

Connected vehicles (such as cars, drones, etc.) attract increasing attention in the community of the Internet of Things. A distributed network of vehicles such as a vehicular ad-hoc network (VANET) can easily be turned into an infrastructure-less self-organizing traffic information system, where any vehicle can participate in collecting and reporting useful information. As the number of vehicular networks continue to grow and now create giant networks (with diverse hierarchical structures and node types), vehicular interactions are becoming more complex [1]. This complexity is further exacerbated by the time-space relationships between vehicles. The intrinsic mobility of the vehicles on the roads leads to highly dynamic and evolutionary topologies. Concurrently, in terms of advanced communication technologies, ultra dense cellular deployments are leading to larger than ever communications among vehicle units (vehicles to infrastructures and infrastructures to infrastructures) while the Device-to-Device (D2D) based vehicular-to-vehicular (V2V) communications generate a complex hybrid communication network [2], [3].

Naturally, vehicles are deployed where human activities occur. Confinement of human settlement in areas limited in size is the foundation of the long-standing Central Place
Theory (CPT) which assumes the existence of regular spatial patterns in regional human organizations. Similarly, cities reflect a statistical self-similarity or a hierarchy of clusters [4]. The towns are split in neighborhoods, each neighborhood is organized in quarters then blocks separated by streets. Such self-similarity is present in traffic too. Figure 1 illustrates the spatial patterns appearing in the traffic in Minneapolis.

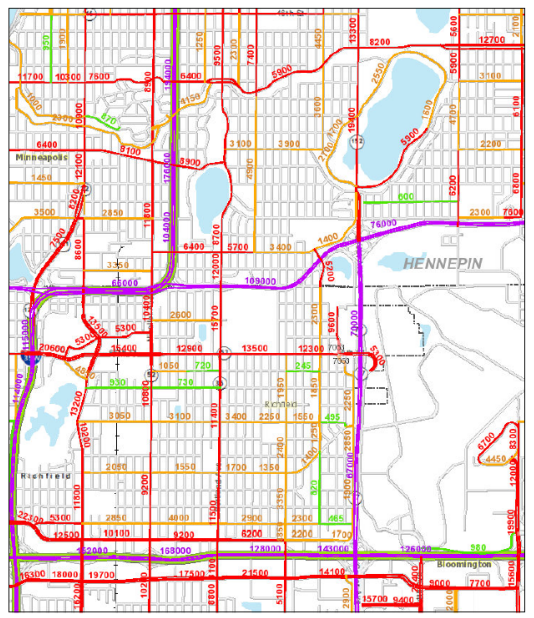

Figure 1: Minneapolis traffic snapshot

The concept of self-similarity is particularly important. In mathematics, a self-similar object, or fractal, is an object which shows strong similarity with smaller parts of itself (i.e. the whole has the same shape as one or more of the parts) [5]). The models of spatial repartition of population following a fractal (and sometimes hyperfractal) distribution have been recently introduced in order to give a more realistic description of interactions that arise in an urban ad-hoc wireless network [6], [7].

Disseminating information in a network is typically done in a broadcast-oriented way [8]. Broadcasting schemes are particularly adequate for vehicular networks due to the mobility of the cars, which implies an ongoing evolving topology [9]. In broadcast protocols, the advantage stands in the fact that the vehicles do not require the knowledge of a specific destination location or its relevant route. This eliminates the complexity of route discovery, address resolution and the previously mentioned topology management with mobility. 
In this context, the objective of the paper is to evaluate the time necessary for a piece of information to propagate in an urban vehicular wireless environment where the population of vehicles is distributed according to a hyperfractal pattern that captures the self-similarity of the topology of the traffic.

Our result: We will prove that the average broadcast time in a hyperfractal setup behaves as $n^{1-\delta}$ times a slowly varying function, where $\delta$ depends on the precise fractal dimension. We prove our model fits real cities traffic using available data sets.

In the past decades, the research community has modeled locations of nodes in a network by extensively using Poisson Point Process (PPP). The seminal work of [10] has enriched the community knowledge on the achievable limits of capacity. In making use of the delay tolerant property of ad-hoc networks, a remarkable work has been done in [11], where a thorough analysis is provided for the broadcast time in a delay tolerant network.

Our hyperfractal topology has been recently introduced in [7] and [12] yet these first works regard only the static case. In contrast, this paper studies a dynamic aspect. Interestingly, the propagation model generates a Delay Tolerant Network (DTN). The network is intermittently interconnected as the spatial repartition of the mobile nodes and the mobility may force packets to "wait" for a vehicle to arrive and receive the packet.

Information dissemination in delay tolerant networks is a long studied problem in vehicular communications as highlighted by the authors of [13] which give an overview of the existing works together with the benefits and weak points. In [14], the authors propose an effective information dissemination algorithm that requires the existence of city maps and GPS traces.

\section{SySTEM MODEL}

\section{A. Hyperfractals}

Cities are hierarchically organized [4]. The centers which form this hierarchy have many elements in common in functional terms and repeat themselves across several spatial scaling. In this sense, districts of different sizes at different levels in the hierarchy have a similar structure. Cities are organized in self-similar structures and represent good candidates for being modeled using fractal geometry.

We propose to use a model focused on the self-similarity of the topology of the traffic, a model that is not in the extremes of the regularity or randomness, but has a behavior similar to a fractal set. By definition, a fractal set has a dimension smaller than the Euclidean dimension of the embedding vector space; it can be arbitrary smaller, [6], [15]. The proposed model is not a fractal but a hyperfractal, in the sense that the dimension is higher than the dimension of the euclidean space. Informally, the hyperfractal model is a Poisson shot model which supports a measure with scaling properties.

Initially, the map is assumed to be the unit square. The support of the population is a grid of streets with an infinite resolution. An example is displayed in Figure 2a. In the first stage the lines forming level 0 are drawn in thick black. In the second stage, each of the four areas obtained is again considered as an independent map with a specific scaling and the lines of level 1 are drawn in thinner black. The process is further continued in a similar manner in the third stage, where each of the 16 areas are again split by 16 crossed drawn in very thin black lines. The split factor of 2 in each dimension is chosen as example yet one can imagine different split factors in each dimension (e.g., 3, 4, etc.).

\section{B. Hyperfractal Mobile Node Distribution}

We now introduce the hyperfractal model for mobile nodes. For completeness we remind briefly the static model studied in our previous works [7], [12].

The map contains $n$ mobile nodes. The process of assigning points to the lines is performed recursively, in iterations, similar to the process of obtaining the Cantor Dust [5]. The two lines of level 0 form a central cross which splits the map in exactly 4 quadrants. We denote by probability $p$ the probability that the mobile node is placed on the cross according to a uniform distribution and $q=1-p$ the complementary probability. With probability $q / 4$, the mobile is placed in one of the four quadrants. The assignation procedure recursively continues in each quadrant and it stops when the mobile node is assigned to a cross of a level $m \geq 0$. A cross of level $m$ consists of two intersecting segments of lines of level $m$ and each segment of the cross is considered to be a segment of level $m$.

A street of level $H$ consists of the union of consecutive segments of level $H$ in the same line. The length of a street is the length of the side of the map. Figure $2 b$ shows the population obtained in the street assignment process after 4 iterations. As one can easily notice, the population density decays with the street level.

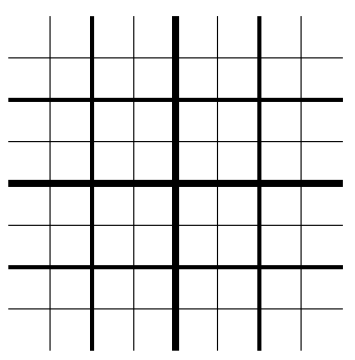

(a)

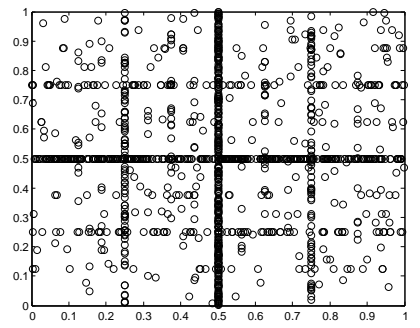

(b)
Figure 2: (a) Hyperfractal map support; (b) Hyperfractal, $d_{F}=$ $3, n=1,200$ nodes;

Taking the unit density for the initial map, the density of mobile nodes in a quadrant is $q / 4$. Let $\lambda_{H}$ be the density of mobile nodes assigned on a street of level $H$. It satisfies:

$$
\lambda_{H}=(p / 2)(q / 2)^{H}
$$

The measure (understood in the Lebesgue meaning) which represents the actual density of mobile nodes in the map has strong scaling properties. The most important one is that the 
map as a whole is identically reproduced in each of the four quadrants but with a weight of $q / 4$ instead of 1 . Thus the measure has a structure which recalls the structure of a fractal set, such as the Cantor map [5]. A crucial difference lies in the fact that the fractal dimension here, $d_{F}$, is in fact greater than 2, the Euclidean dimension. Indeed, considering the map in only half of its length consists into considering the same map but with a reduced weight by a factor $q / 4$. One obtains:

$$
\left(\frac{1}{2}\right)^{d_{F}}=q / 4 \quad \text { thus } \quad d_{F}=\frac{\log \left(\frac{4}{q}\right)}{\log 2}>2
$$

This property can only be explained via the concept of measure.

It is said that a street is busy if the street contains at least one mobile node. The following lemma will play an important role in the proof of our main results:

Lemma 1. The average number of busy streets is asymptotically equivalent to $n^{\delta} \frac{-\Gamma(-\delta)}{\log (2 / q)}$ with $\delta=\frac{\log 2}{\log (2 / q)}$ and where $\Gamma($. is the Euler "Gamma" function. [16].

The proof can be found in the extended version of this paper

Notice that when $p \rightarrow 0$ then $d_{F} \rightarrow 2$ and the measure tends to the uniform measure in the unit square. In other words, a hyperfractal with an asymptotic value of $d_{F}=2$ is a uniform Poisson point process. In this case the number of busy streets tends to $n$, since in an uniform Poisson point process nodes are east-west or north-south aligned with probability zero. This is well reflected in the result above since one would obtain $\delta \rightarrow 1$.

The mobiles move on the lines that are the support of the hyperfractal map. When a node reaches a boundary, it reenters the map from the same point, following a billiard mobility.

Initially, for the sake of simplicity, the speed of the mobiles is considered to be constant and identical, $v$, no matter the level and the density of the nodes on the lines. In reality, the values of speed vary in certain intervals. As our analysis is focused on upper and lower bounds, the variation will not impact the order of magnitude. The case of variable speed will be discussed later, in Section $\mathrm{V}$ where we show that the bounds are validated for variable speed case as well. Due to space limit, we will not analyze the case where some streets are congested and thus speed up the broadcast as some nodes are blocked in intersections.

\section{Canyon Effect}

The analyzed network model is an urban vehicular network that can be made realistic by capturing a well-known phenomenon: the canyon effect [17]. The canyon propagation model implies that the signal emitted by a mobile node propagates only on the street where it stands on. If the network was static, considering the given construction process, the probability that a mobile node is placed in an intersection goes to zero when the street width goes to zero and nodes positioned on two different streets are never able to communicate. Notice that when a street has positive width, the intersection width is negligible compared to the street length and the network will still be partitioned. The connectivity of the network is thus ensured through the mobility of the nodes, leading to a scenario of a delay tolerant network.

\section{Broadcast algorithm}

A feasible approach to forward a packet of information from a source to a destination in the absence of any predictive knowledge on the node movement is an epidemic routing. In this case, when the traffic is low, epidemic routing can achieve an optimal delivery delay at the expense of increased use of network resources. The considered broadcast protocol is a single-hop broadcast meaning that each vehicle carries the information while traveling, and this information is transmitted to the other vehicles in its one-hop vicinity (nearest neighbors of the "infected" node) during the next broadcasting cycle. This single-hop broadcasting protocol relies heavily on the mobility of the vehicles for spreading information.

In this paper, as we primarily seek to understand the limit of the propagation speed, we do not consider other detailed aspects of the broadcast protocol, such as packet collisions. At time $t_{0}=0$ only one node, called "source", holds the packet. At time $t>t_{0}$, the population of nodes is split among nodes that have received the packet, called infected nodes, and nodes that have not yet received the packet, called healthy nodes.

The measured broadcast time represents the time needed for the contamination of the entire network, starting from a single random source.

\section{MAIN RESULTS}

Throughout the following analysis, without lack of generality, we only consider streets which are busy streets as per Section II-B.

\section{A. Upper Bound}

Remark: There are $2^{H}$ streets of level $H$ intersecting each of the streets forming the central cross.

We denote by $I\left(n_{i}, n_{j}\right)$ the average time that a packet takes to jump from one street containing $n_{i}$ nodes to an intersecting street containing $n_{j}$ nodes, assuming all nodes on the first street carry the packet. In fact, it is sufficient to assume that the closest nodes to the intersection carry the packet.

In the following, for sake of generality, we deal with the case where the nodes of interest, $x$ and $y$, are placed on perpendicular lines of respective depths, $H_{a}$ and $H_{b}$. Denote by $T(x, y)$ the time needed for a packet in a broadcast initiated by node $x$ to reach node $y$.

Definition 1. The direct route uses the streets that embed the nodes $x$ and $y$, and contains the intersection between these two streets.

Definition 2. A diverted route between nodes $x$ and $y$ is a route that employs four segments and three intersections.

As an example, in Figure 3, the direct route is drawn in red dotted line and the diverted route is drawn in continuous red line and continuous blue line. 


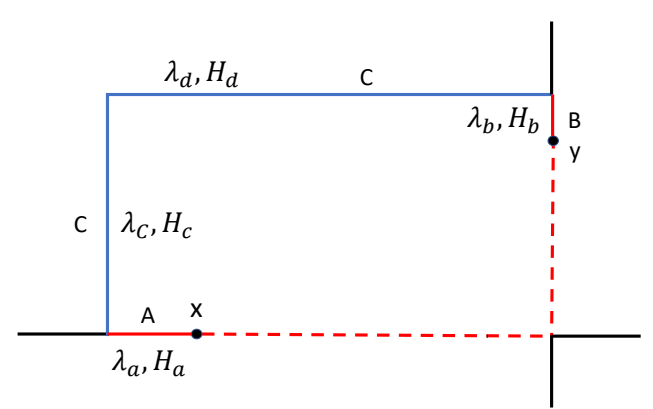

Figure 3: Direct route versus directed route

Lemma 2. Assume that the street of level $H_{a}$ holding node $x$ contains $n_{a}$ nodes, and the street of level $H_{b}$ holding node $y$ contains $n_{b}$ nodes. Given a transmission hop time $h$, the bound in the direct route is:

$$
T(x, y) \leq h n_{a}+h n_{b}+I\left(n_{a}, n_{b}\right) .
$$

Proof. To prove the formulae, let us look at the setup illustrated in Figure 3. In the direct route case it is assumed that the packet hops from node $x$ towards the intersection with the street holding the node $y$ (dashed, red line). The maximum number of hops is $n_{a}$, thus it takes at most $h n_{a}$ time units. The packet turns on the intersection in time $I\left(n_{a}, n_{b}\right)$ and then proceeds towards node $y$ in at most $h n_{b}$ time units.

Lemma 3. Consider the diverted route containing two additional streets of level $H_{c}$ and level $H_{d}$, respectively perpendicular to the street holding $x$, and perpendicular to the street holding $y$, and containing respectively $n_{c}$ nodes and $n_{d}$ nodes. Assuming $n_{c}$ and $n_{d}$ strictly positive, in the diverted route, the bound becomes:

$$
\begin{aligned}
T(x, y) \leq & h\left(n_{a} L\left(H_{c}\right)+1\right)+h\left(n_{b} L\left(H_{d}\right)+1\right) \\
& +I\left(n_{a}, n_{c}\right)+I\left(n_{b}, n_{d}\right)+I\left(n_{c}, n_{d}\right) \\
& +h n_{c}+h n_{d}
\end{aligned}
$$

where $L\left(H_{i}\right)$ is the distance from a node to the intersection with a street of level $H_{i}$.

Proof. Let us again look at the setup illustrated in Figure 3. It is assumed that, instead of taking the direct route as expressed in inequality (3), the packet is diverted into the street of level $H_{c}$, then to the street of level $H_{d}$ before being delivered on the street of node $y$ (straight, red line). The quantity $h\left(n_{a} L\left(H_{c}\right)+1\right.$ and $h\left(n_{b} L\left(H_{d}\right)+1\right)$ is the time necessary for the packet to propagate from $x$ to the intersection with the street of level $H_{c}$ through hop-by-hop propagation, and similarly, the time necessary for the packet to propagate from the intersection of the street of level $H_{d}$ to node $y$. The average number of nodes between $x$ and the streets of level $H_{c}$ is $n_{a} L\left(H_{c}\right)$, due to the uniform node distribution on the interval. Even in considering the node mobility and the time difference between each hop, the mean remains the same since the distribution of nodes remains uniform on the interval. The additive term +1 in the final result comes from the fact that the closest node moving towards the intersection may be located beyond the intersection.

The following lemma gives an estimate of the packet turn time at an intersection.

Lemma 4. For all $n_{i}, n_{j} \in \mathbb{N}^{*}$, the following inequality holds:

$$
I\left(n_{i}, n_{j}\right) \leq \frac{1}{v\left(n_{i}+n_{j}\right)} .
$$

Proof. Let $\Delta$ be the distance between an intersection and a node moving toward the respective intersection. It is assumed, without loss of generality, that the packet originally progresses on an East-West street and that the intersection stands at abscissa $z \in[0,1]$.

Let $g>0$ be the distance toward the intersection of the closest node moving toward the intersection. An upper bound on the probability that $\Delta>g: \Delta$ is greater than $g$ when no node is either in the interval $[z-g, z]$ and is not moving toward the right, or is in the interval $[z, z+g]$ and is moving toward the left. The probability of such an event is upper bounded by $(1-g)^{n}$, in fact it is exactly this expression when $z \in[g, 1-g]$, omitting border effects.

The car at distance $\Delta$ reaches the intersection in $\frac{\Delta}{v}$ time units. At this time the car can transmit the packet to the closest car on the North-South street. Now, merging the problem over the two streets together, the probability that the time for the packet to turn to be larger than $g / v$ is equal to the probability that no car on the East-West street and on the North-South street reaches the intersection before $g / v$ time units, which is upper bounded by $(1-g)^{n_{i}}(1-g)^{n_{j}}$. Thus

$$
v I\left(n_{i}, n_{j}\right) \leq \int_{0}^{1}(1-g)^{n_{i}+n_{j}} d g=\frac{1}{n_{i}+n_{j}} .
$$

The following technical result gives the probability that a street is busy.

Lemma 5. In a hyperfractal with $n$ nodes and $d_{F}>2$, the probability that a street $i$ of level $H(n)$ with $H(n)=$ $\left\lceil\frac{\log \left(n^{1-\epsilon} p / 2\right)}{\log (2 / q)}\right\rceil$ is empty is smaller than $e^{-(q / 2) n^{\epsilon}}$.

Proof.

$$
\mathbb{P}\left(n_{i}=0\right)=\left(1-\lambda_{H(n)}\right)^{n}<e^{-n \lambda_{H(n)}} \leq e^{-(q / 2) n^{\epsilon}}
$$

The following theorem gives the upper bound on the broadcast time and proves that it grows as $n^{1-\frac{1}{d_{F}-1}}$, showing that the growth is sub-linear and only depends on the fractal dimension.

Definition 3. We define by $T_{n}(x, y)$ the time necessary for a packet transmitted in a broadcast initiated by node $x$ to arrive at node $y$. We define by $\mathbf{E}\left[T_{n}(x, y)\right]$ the average broadcast time between all fixed $(x, y)$ pairs. 
Theorem III.1. Consider a network with $n$ mobile nodes in a hyperfractal setup with fractal dimension $d_{F}>2$, transmission hop time $h$ and constant speed of vehicles $v$. Let $x$ and $y$ be two nodes on perpendicular streets. When $n \rightarrow \infty$ the average broadcast time satisfies:

- (i) in the direct route scenario

$$
\mathbf{E}\left[T_{n}(x, y)\right] \leq h p n+\frac{1}{v} ;
$$

- (ii) in the diverted route scenario, for all $\epsilon>0$

$$
\mathbf{E}\left[T_{n}(x, y)\right] \leq 2 h p n^{1-\delta+\epsilon}\left(\frac{2}{p}\right)^{\delta}+\frac{4}{q} h n^{\frac{\epsilon}{\delta}}+\frac{3}{v}+O\left(n e^{-\frac{q}{2} n^{\epsilon}}\right)
$$

where $\delta=\frac{1}{d_{F}-1}$.

Proof. As we look for the upper bound, the inequality in the direct route (eq.(6)) comes directly from $\mathbf{E}\left[n_{a}+n_{b}\right]<p n$. The term $\frac{1}{v}$ is the upper bound of $I\left(n_{i}, n_{j}\right)$ thus maximizing the sum.

The diverted route scenario again follows Figure 3. Let us take $H_{c}=H_{d}=H(n)$ with $H(n)=\left\lceil\frac{\log \left(n^{1-\epsilon} p / 2\right)}{\log (2 / q)}\right\rceil$ as per (5), thus:

$$
\frac{\log \left(n^{1-\epsilon}(p / 2)\right)}{\log (2 / q)}-1 \leq H(n) \leq \frac{\log \left(n^{1-\epsilon}(p / 2)\right)}{\log (2 / q)}+1 .
$$

We have:

$$
\mathbf{E}\left[n_{c}\right]=\mathbf{E}\left[n_{d}\right] \leq n^{\epsilon} \frac{2}{q} .
$$

Meanwhile, let us take as diverted route the closest street of level $H(n)$ from node $x$ since this street is busy with probability higher than $e^{-(q / 2) n^{\epsilon}}$. In this case:

$$
L(H(n)) \leq 2^{-H(n)} \leq 2 n^{-\delta(1-\epsilon)}\left(\frac{2}{p}\right)^{\delta} .
$$

Consequently:

$$
\frac{p}{2} n 2^{-H(n)} \leq p n^{1-\delta+\delta \epsilon}\left(\frac{2}{p}\right)^{\delta}
$$

and the result is obtained by changing the value of $\epsilon$ in $\epsilon / \delta$.

The term $O\left(n e^{-(q / 2) n^{\epsilon / \delta}}\right)$ comes from the case when either $n_{c}=0$ or $n_{d}=0$ which arrives with probability $e^{-(q / 2) n^{\epsilon}}$. In this case, we know that $T_{n}(x, y) \leq h p n+\frac{1}{v}$ thus the contribution to $\mathbf{E}\left[T_{n}(x, y)\right]$ is $O\left(n e^{-(q / 2) n^{\epsilon}}\right)$.

Remarks: (i) As $\epsilon$ becomes smaller, the convergence of eq. (7) is slower. (ii) The quantity $\delta$ is strictly less than 1 $(\delta<1)$ and tends to 1 when $d_{F} \rightarrow 2$. (iii) In fact the term $3 / v$ is far too high while its weight in the sum should not be of high importance. Using Lemma 5, the term can be replaced by $\frac{2}{v n}+\frac{1}{v n^{\epsilon / \delta}}$. Notice that the optimal value of $\epsilon$ is of order $\log \log n / \log n$ which does not reach negligible values as long as $n \ll 1 / h v$ and leads to the global estimate $\mathbf{E}\left[T_{n}(x, y)\right]=$ $\mathcal{O}\left(n^{1-\delta} \log n\right)$.

Definition 4. The average broadcast time $T_{\text {broadcast }}$ is the average of all source-destination pairs $(x, y)$ of $\mathbf{E}\left[T_{n}(x, y)\right]$.
Corollary 1. When $n \rightarrow \infty$, the average broadcast time as the average over all sources $x$ satisfies:

$$
T_{\text {broadcast }}=O\left(n^{1-\delta} \log n\right)
$$

\section{B. Lower Bound}

The intuition behind the lower bound of the average broadcast time comes from the fact that the highest weight in the broadcast time is taken by the time that the packet hops on the main cross, where the density of mobile nodes is considerably higher than on the subsequent levels.

Theorem III.2. Consider a network with $n$ mobile nodes in a hyperfractal setup with fractal dimension $d_{F}>2$, transmission hop time $h$, constant speed of vehicles $v, \delta=$ $\frac{1}{d_{F}-1}$. The average broadcast time satisfies:

$$
T_{\text {broadcast }} \geq \frac{p^{3}}{2} h n^{1-\delta} \frac{\log (2 / q)}{-\Gamma(-\delta)}
$$

Proof. The broadcast time verifies:

$$
T_{\text {broadcast }}=\frac{1}{n^{2}} \sum_{\mathbf{E}}\left[T_{n}(x, y)\right]
$$

when $(x, y)$ are all the possible pairs of two nodes in the hyperfractal.

We denote by $\mathcal{H}$ the set of nodes on the horizontal segment belonging to the central cross and $\mathcal{V}$ the set of nodes on the vertical segment of the central cross.

As we compute the sum only over the terms on the central cross,

$$
T_{\text {broadcast }} \geq \frac{1}{n^{2}}\left(\sum_{\substack{x \in \mathcal{H} \\ y \in \mathcal{V}}} \mathbf{E}\left[T_{n}(x, y)\right]+\sum_{\substack{x \in \mathcal{V} \\ y \in \mathcal{H}}} \mathbf{E}\left[T_{n}(x, y)\right]\right)
$$

as the number of the terms in the sum in (11) is lower than the total number of terms in the sum in (10).

Since the packet must leave the street of node $x$, it must at least run on a distance $L(x)$ which is the average distance from node $x$ to the closest busy perpendicular street. The same holds for reaching nodes $y$. In other words, the following inequality holds:

$$
\mathbf{E}\left[T_{n}(x, y)\right]>h p n \mathbf{E}[L(x)] .
$$

Assume that the node $x$ is on the East-West segment of the central cross. The average distance to the closest North-South busy street is larger than $\frac{1}{2 \mathbf{N S}_{n}}$, where $\mathbf{N S}_{n}$ is the random variable expressing the number of busy North-South streets in presence of $n$ mobile nodes. Therefore, $E[L(x)] \geq \mathbf{E}\left[\frac{1}{2 \mathbf{N S}_{n}}\right]$. The lowest value would be obtained if the busy North-South streets were equally spaced.

By Lemma 1 , one has $E\left[\mathbf{N S}_{n}\right]=\frac{1}{2} B_{n}$. Furthermore $E\left[\frac{1}{\mathbf{N S}_{n}}\right] \geq \frac{1}{E\left[\mathbf{N S}_{n}\right]}$ by convexity of the hyperbolic function. Thus by referring to Lemma 1 :

$$
\mathbf{E}\left[T_{n}(x, y)\right]>\frac{h p n}{B_{n}}=h p n \frac{\log (2 / q)}{-\Gamma(-\delta)} .
$$


Using the fact that $\mathbf{E}[|\mathcal{H}||\mathcal{V}|]=(n-1) n p^{2} / 4$ terminates the proof.

Corollary 2. The average broadcast time when $n \rightarrow \infty$ satisfies:

$$
T_{\text {broadcast }}=\Omega\left(n^{1-\delta}\right)
$$

Combining the upper bound in Corollary 1 and the lower bound of Corollary 2, one obtains the matching bound that the average broadcast time behaves as $n^{1-\delta}$ times a slowly varying function, where $\delta$ depends on the precise fractal dimension:

Corollary 3. The average broadcast time when $n \rightarrow \infty$ satisfies:

$$
\lim _{n \rightarrow \infty} \frac{\log T_{\text {broadcast }}}{\log n}=1-\delta=1-\frac{1}{d_{F}-1} .
$$

\section{Information Teleportation}

In a hyperfractal, the broadcasted packet follows either a direct route or a diverted route. The diverted route case leads to the existence of new contagions on the lines of level $H_{c}$ and $H_{d}$. This is what we call "information teleportation" phenomenon as the new contagions are not due to a source on lines $H_{c}$ or $H_{d}$ spreading its packet in a hop by hop manner but is due to routing the packets through intersections. The phenomenon will be visually illustrated by experiments in Section V.

The teleportation phenomenon allows an acceleration of the broadcast time. The acceleration itself is a self-similar phenomenon and takes places recursively: propagation on level $H_{i}$ is accelerated by teleportation coming from lines $H_{i+1}, H_{i+2}, H_{i+3}$ and so on.

In a hyperfractal with teleportation effect, the broadcast time evolves as $\mathcal{O}\left(n^{1-\delta} \log n\right)$ according to Corollary 1 . To consider a network with the absence of teleportation is to consider the direct route case in Theorem III.1. In such a network, the broadcast time scales linearly as $\mathcal{O}(n h)$. The two regimes are illustrated in Figure 4.

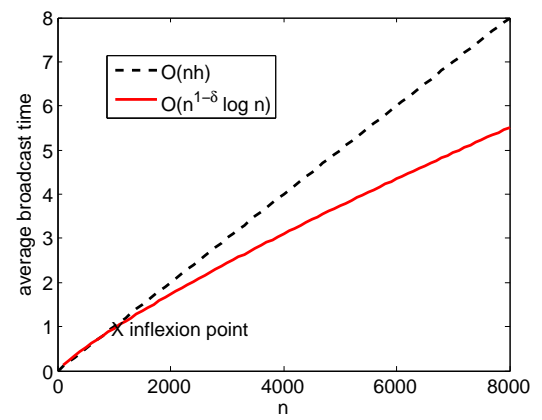

Figure 4: Broadcast time evolution in hyperfractal vs linear regime: Inflexion point

The teleportation phenomenon arises after the linear characteristic overtakes the one for $O\left(n^{1-\delta} \log n\right)$. The inflexion point where teleportation arises verifies $n h=\mathcal{A} n^{1-\delta} \log n$, where $\mathcal{A}$ is a constant.

Let us look at an example of broadcast speed up that occurs due to information teleportation. In a hyperfractal setup, consider an infected source on a line of level $H_{i}=0$. In each time slot $h$, two more nodes get infected and become themselves sources. In the absence of teleportation, therefore, the number of infected points increases linearly with the hop time. In the presence of intersections with lines of levels $H_{i}>0$, new "outbreaks of infection" arise at time stamps of $\frac{1}{v n\left(\lambda_{0}+\lambda_{H_{i}}\right)}$.

Figure 5 shows graphically the evolution. This is an upper bound as not all the teleportation contagions generate a speed up; the nodes can be infected from neighboring contagions by simple hop by hop propagations.

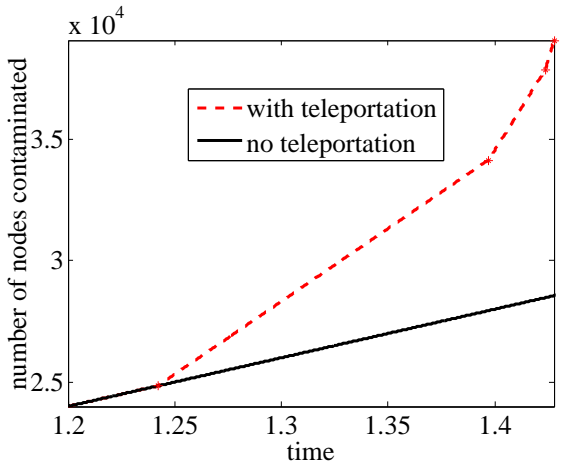

Figure 5: Propagation speed up on the line of level 0

\section{GENERALIZATION AND VALIDATION OF THE HYPERFRACTAL MODEL}

In this section, we show that the hyperfractal model fits real cities. Let us describe the procedure that computes the fractal dimension of a city using the spatial traffic density criterion.

The spatial traffic density criterion is about the statistic of the street densities in the map.

In a hyperfractal, the cumulated length of the street up to level $H$ is $2^{H+1}-2$. At this level, $H$, the density of the nodes on the streets is $\frac{p}{2}\left(\frac{q}{2}\right)^{H}$.

In the general setting we consider as a single street an alignment of consecutive segments whose density from the less dense segment to the densest segment does not vary more than by a factor $F>1$. We call the density of the street the average density of its segment. In a pure hyperfractal city model we have $F=1$. Next, we rank the streets in decreasing order of density: $\lambda_{1} \geq \lambda_{2} \geq \ldots \geq \lambda_{i} \geq \ldots$. Denote by $l_{i}$ the length of the $i$ th street.

We define the density as a function of the cumulated distance $l, \lambda(l)$ as follows: if $\sum_{i}^{k-1} l_{i}<l \leq \sum_{i}^{k} l_{i}$ then $\lambda(l)=\lambda_{k}$. It can be expressed as:

$$
\lambda(l)=\Theta\left(l^{1-d_{F}}\right)
$$

In order to illustrate how the hyperfractal fits vehicles distribution on streets, we present here data fitting results using public measurements [18]. Figure 1 shows the snapshot of a traffic flow map displaying the average annual weekday traffic in a neighborhood of Minneapolis. By applying the described fitting procedure and using (14), the estimated fractal dimension for Minneapolis is $d_{F}=2.9$. In Figure 6 we 
illustrate the fitting of the data for the density repartition function, proving how traffic flow maps are transformed into hyperfractals. The fitting becomes more accurate with the increase of number of terms in the density repartition function.

Additional criteria such as time interval intersection criterion are presented in the extended version of the paper [16].

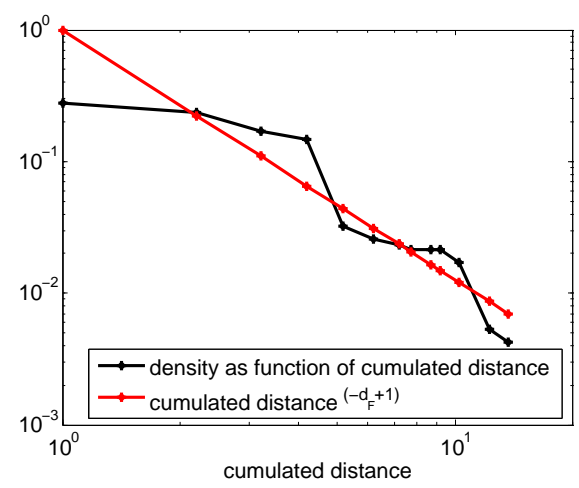

Figure 6: Model fitting

\section{Simulations}

The map length is the unit and a scaling is performed in order to respect the scaling of real cities as well as communication and motion parameters. In that sense, it is considered that the unit represents $10 \mathrm{kms}$ of distance in a real scale. Respecting this scaling, the value of speed in the simulations is $v=10^{-3}$. The hop duration is $=1 \mathrm{~ms}$, in line with most protocols used for vehicular communications.

Throughout the simulations, the depth of the hyperfractal are restricted to $H=5$.

\section{A. Information spread under hyperfractal model and Teleport- ation phenomenon}

We first study information propagation in a full idealized epidemic broadcast with its specific phenomenons. In a two dimensional uniform Poisson point process, the information packet spreads uniformly as a full disk that grows at a constant rate, which coincides with the information propagation speed, as shown in [11].

Interestingly, in a hyperfractal, the phenomenon is completely different, as a consequence of the canyon effect and the population distribution specific to the new model.

The simulations are performed using the following scenario: a source starts an epidemic broadcast of an information packet at time $t=0$ in a network of 1,200 nodes in a $1 \mathrm{x} 1$ unit square, mobile node speed $v=10^{-3}$, packet transmission time, also called hop time is $h=10^{-3}$. The population of 1,200 nodes is distributed in the map according to a hyperfractal of dimension $d_{F}=5.33$.

Figure 7 shows different stages in the information propagation starting from a random chosen source until the complete contamination of the nodes.

Notice how, although the information propagates along the streets of the network, it does not propagate in a uniform way (e.g., like a growing disk). The propagation follows the repartition of the population, the constraints imposed by the environment (i.e., intersections) and accelerates along the streets (i.e., canyon effect).

Figure 7b shows "the teleportation phenomenon" in a network of $n=1,200$ nodes with $d_{F}=5.33$. Two contagions of infected nodes on the lines of level 0 are highlighted. These areas are not connected to the main infected area on the line on which they originate, the line of level $H=0$. The nodes on these areas are infected by receiving the packet from nodes traveling on perpendicular lines. This generates several areas of contagion. On this line, the packet is spread from all of the contamination sources that have arisen and thus the broadcast is sped up. This is a phenomenon that uniquely characterizes the broadcast in hyperfractal setups.

\section{B. Validation of upper and lower bounds. Average broadcast time}

The experiments are run for numerous values of $n$ starting with $n=300$ and then from $n=500$ up to 5,000 nodes with a step of 500 and $d_{F}=3$ and $d_{F}=5.33$, thus $p=0.5$, and 0.9 respectively.

The formulation used for the upper bound is the expression in equation (4), while for the lower bound the formulation used for validation through simulations is the closed expression $T>$ $h n L(x)$ with $L(x)=\frac{1}{B_{n}}$ and $B_{n}=\frac{n^{\delta} \Gamma(\delta)\left(\frac{p}{2}\right)^{\delta}}{\log (2 / q)}$.

Figure 8 a validates Theorems 2 and III. 2 on the expression of the average broadcast time.

Notice that the lower bound follows with a good approximation the simulation results. Each of the cases shows that the broadcast time increases with the decrease of the number of points, a phenomenon which is captured successfully by the upper bound. The upper bound increases with a higher slope for big fractal dimension (see Figure 8b), converging towards the asymptotic bound of $\mathcal{O}\left(n^{1-\delta} \log n\right)$.

\section{Speed Variation}

The theoretical results are developed under the assumption of constant speed throughout the whole network map. The following experiments show that the bounds hold for the more realistic scenario of variable values of speed.

The first analysis looks at the case where the speed of the nodes is proportional to the level, $v \propto H$. This models the scenario where the speed is lower on crowded streets, due to congestion, and increases with the decrease of density of nodes. More precisely, the nodes on a level $H_{i}$ have the same speed $v_{i} \propto H_{i}$.

The values of speed are chosen such as to represent, in realsetup, $v=36 \mathrm{kmph}$ for $H=0, v=40 \mathrm{kpmh}$ for $H=1$, $v=50 \mathrm{kmph}$ for $H=2, v=60 \mathrm{kmph}$ for $H=3$ and $v=70 \mathrm{kmph}$ for $H=4$. Figure 9a validates both upper and lower bound on the broadcast time. The second analysis looks at the case where the speed is proportional to the inverse of the level, $v=\frac{1}{H}$. This models the scenario where speed is higher on streets with a high level of importance in the city, like highways, and decreases with the street importance, for example, alleys. For this case, the values of speed are chosen 


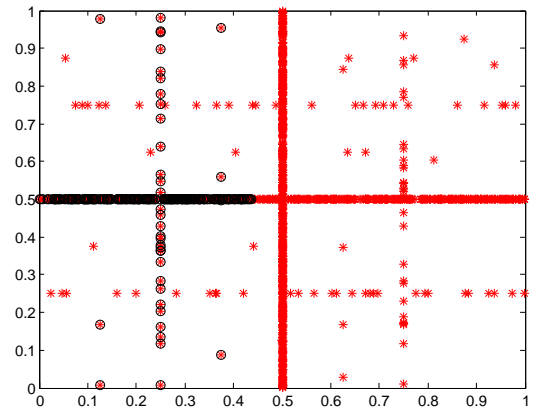

(a) One of the initial stages

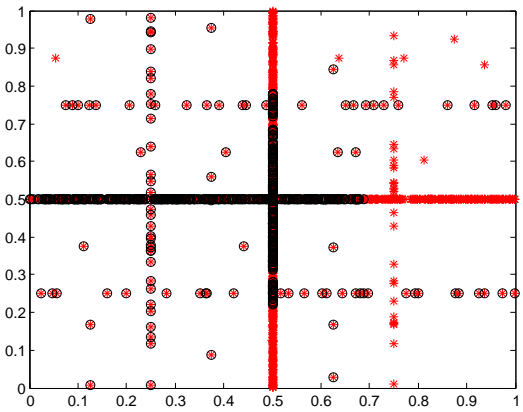

(b) Intermediate stage

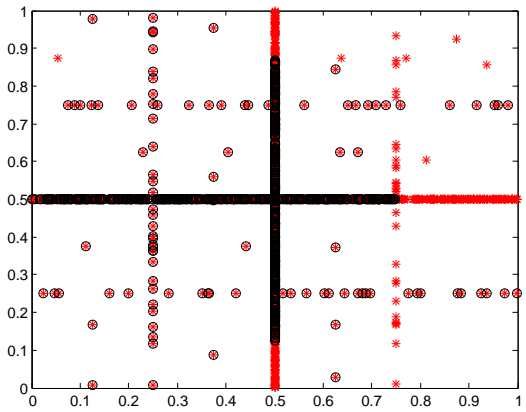

(c) One of the final stages

Figure 7: Snapshots of information dissemination in a Hyperfractal, healthy nodes in red '*', infected nodes in black 'o'

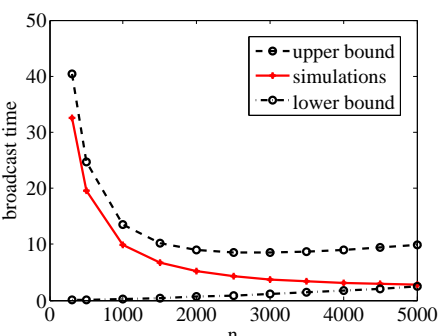

(a)

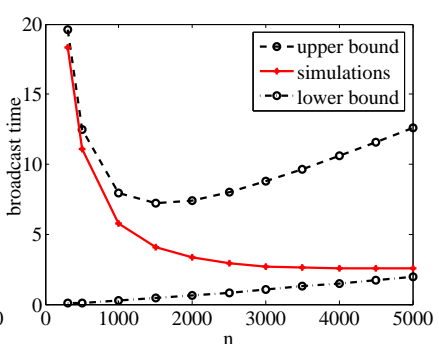

(b)
Figure 8: Broadcast time: simulation vs. theoretical results for (a) $d_{F}=3$, (b) $d_{F}=5.33$

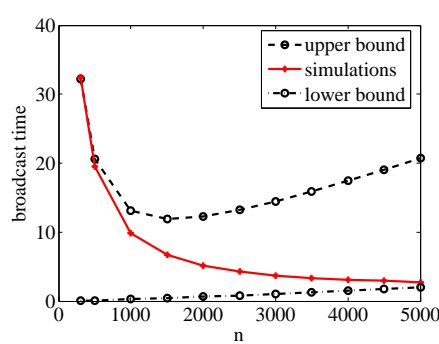

(a) $v_{H} \propto \lambda_{H}, d_{F}=3$

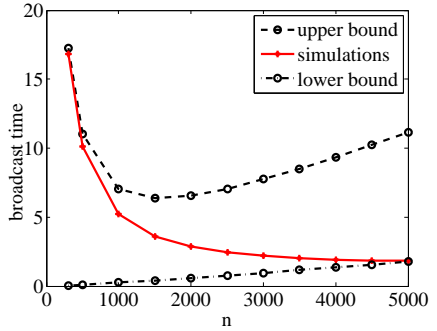

(b) $v_{H} \propto 1 / \lambda_{H}, d_{F}=3$
Figure 9: Speed variation proportional or inversely proportional to the street level

to correspond to real-setup values of: $v=36 \mathrm{kmph}$ for $H=4$, $v=40 \mathrm{kpmh}$ for $H=3, v=50 \mathrm{kmph}$ for $H=2, v=60$ kmph for $H=1$ and $v=70 \mathrm{kmph}$ for $H=0$. Figure $9 \mathrm{~b}$ validates both upper and lower bound on the broadcast time.

\section{CONCLUSIONS}

This paper initiated a characterization of the information propagation speed of of vehicular delay tolerant mobile networks in urban scenario by using a novel model for the traffic topology of the mobile vehicles and by providing matching theoretical upper and lower bounds for such networks. These theoretical bounds both increase our understanding of the fundamental properties and performance limits of vehicular networks in urban environments, and help us optimize the per- formance of specific routing algorithms. The enhanced model captures self-similarity as an environment characteristic and highlights the speedup in propagation due to the teleportation phenomenon. This opens new opportunities in the study of urban vehicular networks. The paper also provides a method for fitting the model to real traffic data.

\section{REFERENCES}

[1] S. Abdelhamid, H. S. Hassanein, and G. Takahara, "Vehicle as a resource (vaar)," IEEE Network, 2015.

[2] M. Gerla, E. K. Lee, G. Pau, and U. Lee, "Internet of vehicles: From intelligent grid to autonomous cars and vehicular clouds," in 2014 IEEE World Forum on Internet of Things (WF-IoT).

[3] E. Schoch, F. Kargl, and M. Weber, "Communication patterns in vanets," IEEE Communications Magazine.

[4] M. Batty, "The size, scale, and shape of cities." Science, 2008.

[5] B. B. Mandelbrot, The Fractal Geometry of Nature, 1983.

[6] P. Jacquet, "Optimized outage capacity in random wireless networks in uniform and fractal maps," in ISIT, June 2015, pp. 166-170.

[7] P. Jacquet and D. Popescu, "Self-similarity in urban wireless networks: Hyperfractals," in Workshop on Spatial Stochastic Models for Wireless Networks, SpaSWiN, May 2017.

[8] R. Chen, W. L. Jin, and A. Regan, "Broadcasting safety information in vehicular networks: issues and approaches," IEEE Network.

[9] C. Boldrini and A. Passarella, Data Dissemination in Opportunistic Networks, 2013.

[10] P. Gupta and P. R. Kumar, "The capacity of wireless networks," IEEE Transactions Information Theory, pp. 388-404, 2006.

[11] P. Jacquet, B. Mans, and G. Rodolakis, "Information propagation speed in mobile and delay tolerant networks," IEEE Transactions on Information Theory, vol. 56, no. 10, pp. 5001-5015, Oct. 2010.

[12] P. Jacquet and D. Popescu, "Self-similar geometry for ad-hoc wireless networks: Hyperfractals," in 3rd Conf. on Geometric Science of Information, 2017.

[13] S. M. Tornell, C. T. Calafate, J. C. Cano, and P. Manzoni, "Dtn protocols for vehicular networks: An application oriented overview," IEEE Communications Surveys Tutorials.

[14] F. Martinez and et al., "Evaluating the impact of a novel warning message dissemination scheme for vanets using real city maps," in NETWORKING 2010.

[15] Wikipedia, "Cantor set - wikipedia, the free encyclopedia," 2016.

[16] P. Jacquet, D. Popescu, and B. Mans, "Information dissemination in vehicular networks in an urban hyperfractal topology," arXiv: 1712.04054 .

[17] F. J. Martinez, C. K. Toh, J. C. Cano, C. T. Calafate, and P. Manzoni, "Realistic radio propagation models (rpms) for vanet simulations," in 2009 IEEE Wireless Communications and Networking Conference.

[18] "Minnesota department of transortation data," https://www.dot.state.mn. us/tmc/trafficinfo/traffic.html, accessed: 2017-11-22. 